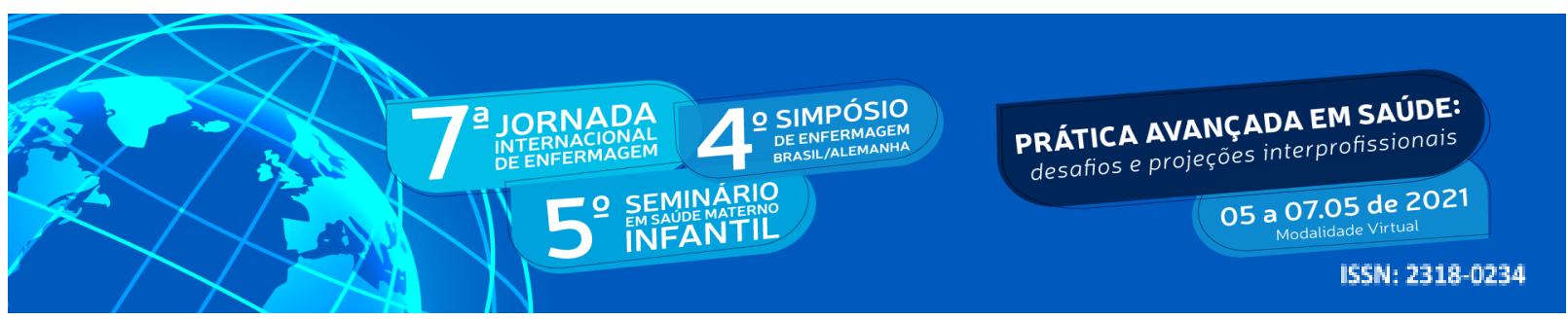

DOI: http://doi.org/10.48195/jie2021-023

\title{
HIPERTENSÃO ARTERIAL E ENVELHECIMENTO: UM RELATO DE EXPERIÊNCIA ${ }^{1}$
}

\section{Malwiani Aparecida Caumo² ${ }^{2}$ Natasha Gazzolla Sporquio ${ }^{3}$; Tereza Cristina Blasi ${ }^{4}$}

\begin{abstract}
RESUMO
Nos últimos anos, houve um rápido aumento do envelhecimento populacional no Brasil. Com isso, percebe-se a existência de uma relação direta e linear entre $\mathrm{o}$ ato de envelhecer com $\mathrm{o}$ desenvolvimento da Hipertensão Arterial (HA). O objetivo desse artigo foi relatar a experiência de acadêmicas do curso de Nutrição na elaboração de um material informativo sobre Hipertensão Arterial no envelhecimento e sua posterior publicação nas redes sociais. Para tanto, a metodologia utilizada foi a criação de um vídeo por meio dos softwares Microsoft PowerPoint e Adobe Premiere. Como resultado, obteve-se um vídeo abordando a temática central da hipertensão relacionando-a com o envelhecimento. Levando-se em conta o que foi observado, foi possível concluir que as redes sociais são plataformas viáveis para a divulgação de informações de promoção à saúde, porém, percebe-se que ainda há limitações desse uso, principalmente quando o público-alvo são os idosos.
\end{abstract}

Palavras-chave: Extensão; Prevenção de Doenças; Saúde do Idoso.

\begin{abstract}
In the last years, there was a fast increase of population aging in Brazil. With this, realize the existence of a direct and linear relation between the act of age with the Arterial Hypertension (AH) development. The objective of this article was reported the experience of Nutrition academics on elaboration of an informational material about Arterial Hypertension in aging and its after publication on social networks. For this, the methodology used was the creation of a video through the softwares Microsoft PowerPoint and Adobe Premiere. As a result, got a video approaching the central theme of hypertension related to aging. Based on what was observed, it was possible to conclude that the social networks are viable platforms for the divulgation of health promotion information, but, realize that there are limitations of this use, mainly when the target public are elders.
\end{abstract}

Key Words: Extension; Prevention of Diseases; Elder Health.

\footnotetext{
${ }^{1}$ Relato de experiência de acadêmicas do Curso de Nutrição da Universidade Franciscana - UFN.

${ }^{2}$ Estudante do Curso de Nutrição e bolsista do PET-Saúde Interprofissionalidade. Universidade Franciscana.

E-mail: malwiani.caumo@ufn.edu.br

${ }^{3}$ Estudante do Curso de Nutrição. Universidade Franciscana. E-mail: natasha.gazzolla@ufn.edu.br

${ }^{4}$ Orientadora. Professora do curso de Nutrição - UFN. Especialista em Terapia Nutricional - UFSC. Mestre em Ciências e Tecnologia de Alimentos - USFM. Coordenadora do grupo Assistência Multidisciplinar Integrada aos Cuidadores de Pessoas com Alzheimer - AMICA . Universidade Franciscana. E-mail: terezacristina@ufn.edu.br
} 


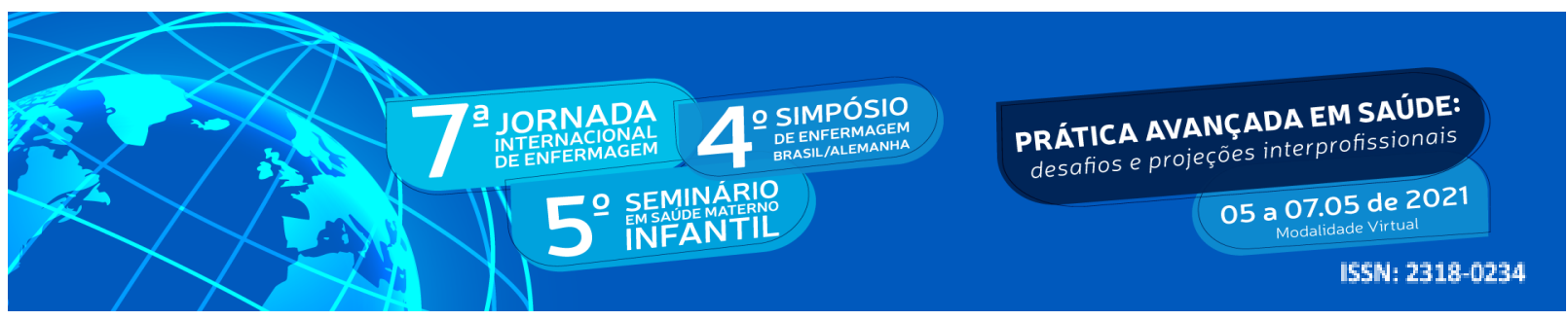

\section{INTRODUÇÃO}

Observa-se no cenário atual, o rápido processo de envelhecimento populacional ocorrido no Brasil e no mundo, o que gera novas demandas sociais e governamentais para garantir os direitos dessa população (BRASIL, 2006; WONG; CARVALHO, 2006). Nascer, crescer e envelhecer são processos naturais e não dependem apenas da condição genética do indivíduo, ou seja, a genética alia-se ao estilo de vida das pessoas (FECHINE; TROMPIERI, 2012).

Além de todas as modificações sociais, pessoais e fisiológicas do envelhecimento, os idosos, no último ano, depararam-se com o isolamento social e com a implementação de várias tecnologias no seu cotidiano. Essa implementação foi positiva, visto que reduziu a sensação de solidão e isolamento desse público, tornando-se evidente a necessidade de incluir os idosos no meio digital (COSTA et al., 2021).

O uso de redes sociais como WhatsApp $^{\circledR}$, Facebook $^{\circledR}$ e Instagram $^{\circledR}$ foi avaliado também como positivo por essa população no que diz respeito ao acesso à informações, seja através de lives ou posts. Isso mostra que os idosos estão em evidência no quesito avanço de práticas digitais e que suas idades não devem ser consideradas como um empecilho (VELHO; HERÉDIA, 2020).

Devido ao aumento da expectativa de vida da população brasileira e do aumento de indivíduos idosos na última década, percebeu-se que há uma associação direta e linear entre o envelhecimento e a prevalência de várias doenças, como por exemplo da Hipertensão Arterial (HA) (SBC, 2016).

A HA atinge $32,5 \%$ da população brasileira, ou seja, 36 milhões de indivíduos adultos no Brasil. Além disso, mais de $60 \%$ dos idosos, ocasionando de forma direta ou indireta $50 \%$ das mortes por doença cardiovascular (DCV). Há vários fatores de risco que influenciam no aumento dos níveis de pressão arterial, sendo eles: fumo, consumo de bebidas alcoólicas, obesidade, estresse, elevado consumo de sal, níveis altos de colesterol e sedentarismo. Ressalta-se também que a doença é herdada dos pais em 90\% dos casos (SBC, 2016). 


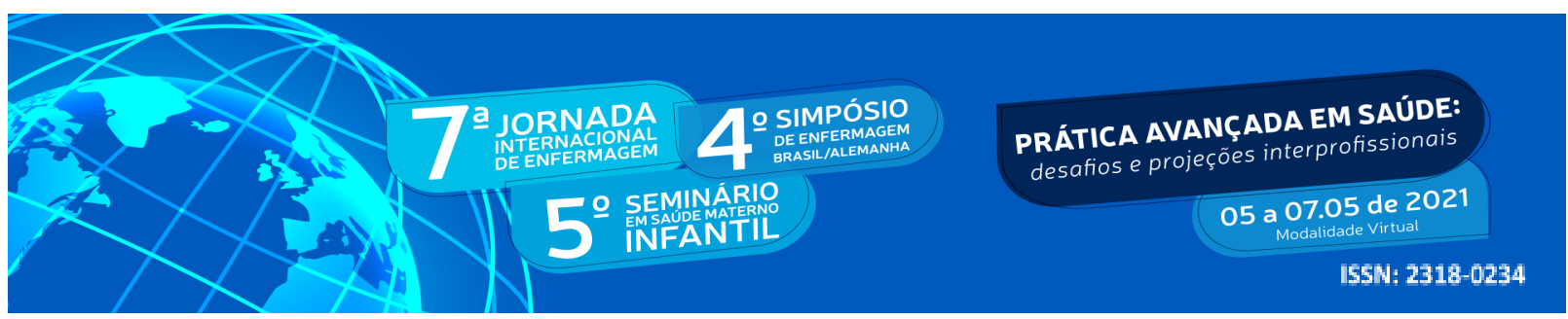

Dessa forma, a HA é uma condição clínica multifatorial caracterizada pela constante elevação da pressão arterial (níveis pressóricos $\geq 140$ e/ou $90 \mathrm{mmHg}$ ) e frequentemente está associada com alterações em órgãos-alvo e distúrbios metabólicos, agravando-se na presença de dislipidemias, Diabetes Mellitus e obesidade (SBC, 2016).

Visto que há uma prevalência significativa de polifarmácia na população idosa (PEREIRA, et al., 2017) e que o tratamento não medicamentoso da HA compreende medidas nutricionais saudáveis e prática de atividades físicas (SBC, 2016), existe a necessidade de buscar alternativas acessíveis a essa população que visem melhorar a sua alimentação e saúde física, consequentemente reduzindo a incidência de HA.

\section{OBJETIVO}

O presente trabalho teve como objetivo relatar a experiência de acadêmicas do curso de Nutrição na elaboração de um material informativo sobre Hipertensão Arterial no envelhecimento e sua posterior publicação nas redes sociais.

\section{METODOLOGIA}

Antes de iniciar-se a elaboração e a edição do material informativo em formato de vídeo, escreveu-se um roteiro tendo como bases principais de pesquisa o livro "Essências em Geriatria Clínica” de Garcia et al. (2018) e a Diretrizes Brasileiras de Hipertensão Arterial (2016).

O material foi elaborado de maneira a propiciar informações mais claras e objetivas, com a finalidade de fornecer maior entendimento acerca do assunto à população-alvo. Por isso, foram utilizados recursos como: imagens ilustrativas, animações, áudios explicativos, legendas e outros.

Para a construção do vídeo foram utilizados os Softwares PowerPoint e Adobe Premiere. O material elaborado foi compartilhado através de redes sociais Instagram $^{\circledR}$ e WhatsApp ${ }^{\circledR}$ pessoais e profisssionais das acadêmicas e da Instituição de Ensino. 


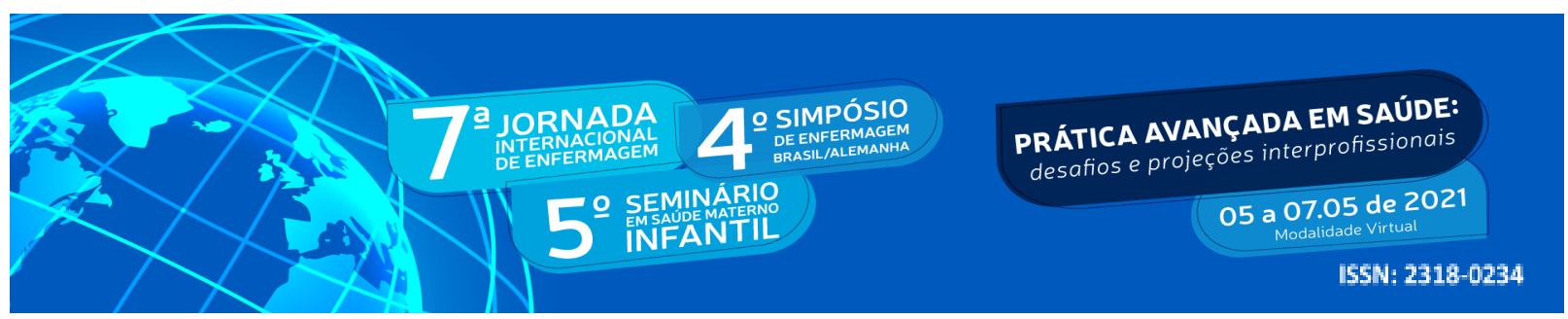

\section{RESULTADOS E DISCUSSÃO}

Como resultado obteve-se um vídeo abordando a temática central da Hipertensão Arterial e relacionando-a com o envelhecimento. A partir disso, os tópicos apresentados no material foram: definição geral da doença com explicações sobre HA, pressão arterial, fatores de risco e fatores ou hábitos que favorecem o desenvolvimento da doença; dados epidemiológicos do Brasil em relação a prevalência e incidência de HA na população idosa; e, por último, explicações sobre a relação do envelhecimento com o desenvolvimento de HA, prevenção e tratamento da doença.

Há estudos que demonstram a importância de propagar informações sobre HA e de investir em ações relacionadas ao controle da doença com a população acometida, proporcionando, assim, uma maior adesão ao tratamento adequado (COELHO, 2015; GONZALEZ, 2016). Por isso, reforça-se o papel da Atenção Primária em Saúde (APS), como meio de se estabelecer formas de educação em saúde. Já que ela representa a principal porta de entrada de muitos indivíduos portadores de HA ao sistema de saúde, dessa forma, possui um importante papel na disseminação e na promoção de práticas que envolvem temáticas voltadas à saúde da população (MACHADO et al., 2016).

O vídeo elaborado foi publicado nas redes sociais $\operatorname{Instagram~}^{\circledR}$ através da página de extensão da instituição de ensino das acadêmicas e no WhatsApp ${ }^{\circledR}$ através de grupos e contatos de professores e acadêmicos, tornando possível ampliar a divulgação do trabalho não somente no estado do Rio Grande do Sul, como também em vários outros estados do Brasil.

Compreende-se que a educação nutricional do idoso tem muita importância como forma de promoção de saúde e prevenção de doenças, visto que a educação nutricional gera mudança de hábitos alimentares nos indivíduos. Isso colabora na prevenção de doenças e no tratamento delas, além de impulsionar a manutenção de práticas alimentares mais saudáveis (BRANDÃO et al., 2010).

Sabe-se também que as redes sociais podem ser utilizadas pelos idosos como fonte de comunicação, estabelecimento de amizades e suporte social em modo virtual. Porém, percebe-se ainda que as redes sociais e outras tecnologias são, de certa forma, distantes das pessoas idosas (CARVALHO, et al.,2014). O idoso usuário que utiliza a internet e as 


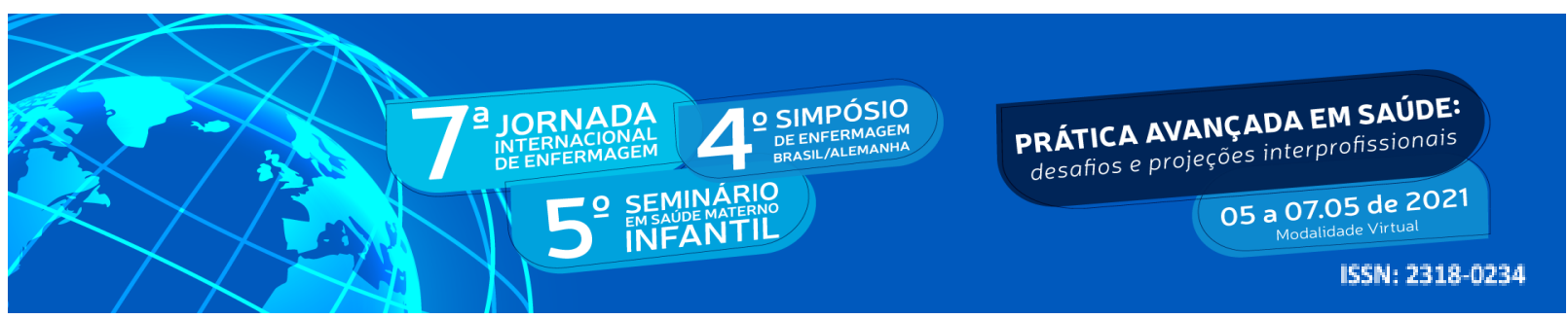

tecnologias relacionadas a ela possuem menos chance de ter depressão, além de manter níveis cognitivos melhores (MIRANDA; FARIAS, 2009).

\section{CONCLUSÃO}

Conclui-se que, utilizar as redes sociais para divulgar informações relacionadas à promoção de saúde em geral e a educação nutricional mostra-se viável, principalmente atualmente, tendo em vista a maior acessibilidade da população às novas tecnologias.

Além disso, destaca-se a importância dos profissionais de saúde em se empoderar de novas estratégias para divulgação de conhecimentos, informações e ações em saúde para a população.

Contudo, ressalta-se a importância de entendimento sobre as limitações do idoso no uso de novas tecnologias e redes sociais, sejam estas limitações provenientes de alguma doença que comprometa a função cognitiva e/ou física, por falta de acesso às tecnologias ou falta de compreensão sobre o uso e o objetivo dessas ferramentas.

Assim, salienta-se a necessidade de mais estudos que avaliem os impactos e as potencialidades da utilização das redes sociais como espaço para a divulgação de informações relacionadas à saúde do idoso.

\section{REFERÊNCIAS}

BRANDÃO, A. L. et al. Educação Nutricional Para Idosos e seus Cuidadores no Contexto da Educação em Saúde. Revista Vittalle, v. 22, n. 1, p. 27-37, 2010.

BRASIL. Ministério da Saúde. Gabinete do Ministro. Portaria n 2.528 de 19 de outubro de 2006. Aprova a Política Nacional de Saúde da Pessoa Idosa. Brasília, 2006. Disponível em: http://bvsms.saude.gov.br/bvs/saudelegis/gm/2006/prt2528 $19 \quad 10 \quad$ 2006.html\#: :text=A\%20 Pol\%C3\%ADtica \%20Nacional $\% 20 \mathrm{do} \% 20$ Idoso,94\%20e\%20Decreto\%20n $\% \mathrm{C} 2 \% \mathrm{BA} \% 201.9$ 48\%2F. Acesso em: 11 mar. 2021.

CARVALHO, G. M. et al. Redes Sociais e Geratividade: a Experiência do Programa Idosos On-line. Revista Estudos Indisciplinares sobre o Envelhecimento, v. 19, n. 3, p. 793-812, 2014. 


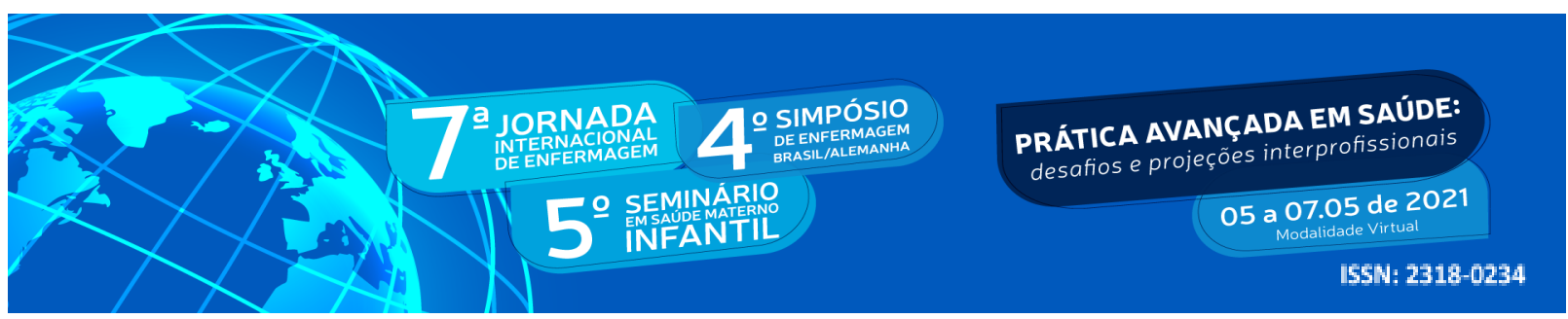

COELHO, R. F. Adesão Terapêutica ao Tratamento da Hipertensão Arterial Sistêmica de uma Unidade da ESF, de Município Mineiro. 2015. 43 f. Trabalho de Conclusão de Curso (Especialização em Atenção Básica em Saúde da Família) - Universidade Federal de Minas Gerais, Governador Valadarez - MG, 2015.

COSTA, D. E. S. et al. A influência das tecnologias na saúde mental dos idosos em tempos de pandemia: uma revisão integrativa. Journal Research, Society and Development, v. 10, n. 2, 2021.

FECHINE, B. R. A.; TROMPIERI, N. O processo de envelhecimento: as principais alterações que acontecem com o idoso com o passar dos anos. Journal Inter Science Place, Ceará, ed. 20, v. 1, art. 7, jan./mar. 2012.

GARCIA, E. et al. Essências em Geriatria Clínica. Porto Alegre: EdiPUCRS, 2018.

GONZALEZ, B. M. Educação e Promoção de Saúde em Hipertensão Arterial na Estratégia de Saúde da Família. 2016. 42 f. Trabalho de Conclusão de Curso (Curso de Especialização em Atenção Básica em Saúde da Família) - Universidade Federal de Minas Gerais, Uberlândia - MG, 2016.

MACHADO, J. C. et al. Análise de três estratégias de educação em saúde para portadores de hipertensão arterial. Revista Ciência \& Saúde Coletiva, v. 21, n. 2, p. 611-620, 2016.

MIRANDA, L. M.; FARIAS, S. F. As contribuições da internet para o idoso: uma revisão de literatura. Revista Interface - Comunicação, Saúde, Educação, v. 13, n. 29, p. 383-394, abr./jun. 2009.

PEREIRA, K.G. et al. Polifarmácia em idosos: um estudo de base populacional. Revista Brasileira de Epidemiologia, v. 20, n. 2, p. 335-344, abr./jun. 2017.

SOCIEDADE BRASILEIRA DE CARDIOLOGIA. $7^{\text {a }}$ diretriz brasileira de hipertensão arterial. Revista Arquivos Brasileiros de Cardiologia, v. 107, n. 3, supl. 3, set. 2016.

VELHO, F. D.; HERÉDIA, V. B. M. O idoso em quarentena e o impacto da tecnologia em sua vida. Revista Rosa dos Ventos Turismo e Hospitalidade, v. 12, p. 1-14, 2020.

WONG, L. L. R.; CARVALHO, J. A. O rápido processo de envelhecimento populacional do Brasil: sérios desafios para as políticas públicas. Revista Brasileira de Estudos de

População, São Paulo, v. 23, n. 1, p. 5-26, jan./jun. 2006. 